\title{
The Odds Ratio: a useful tool in neurosciences
}

\author{
PETER SANDERCOCK \\ From the Department of Clinical Neurosciences, Western General Hospital, Edinburgh, UK
}

SUMMARY The odds ratio is very useful; when used as a measure of association (or to describe the results of a randomised controlled treatment trial) it expresses both the strength and direction of the association (or size and direction of treatment effect). Its $95 \%$ confidence interval estimates the likely degree of sampling error and provides a test of significance at the $5 \%$ level. Authors, when reporting the results of descriptive studies, case-control studies and randomised controlled trials, should consider presenting their results this way rather than by simple significance testing with a chi squared test.

The odds ratio (an unlikely name for a useful tool) has been around for a long time.' Although it was originally used to analyse case-control studies, it has more recently been applied to other research designsdescriptive studies, randomised trials and overviews of randomised trials. Despite its widespread use in medicine, many of the basic textbooks of medical statistics mention the odds ratio only briefly, if at all. The aim of this article is to describe the usefulness and practical application of the odds ratio simply and clearly and with minimal use of the mathematical formulae that appear in articles written by statistician$\mathbf{s}^{1-3}$ (whose complexity may induce sleep, diplopia or tension headache in the average neurological reader).

The first problem to overcome is terminology. The terms rate, risk, risk difference, relative risk (or risk ratio), odds and odds ratio each have precise epidemiological definitions, and cannot be used interchangeably. ${ }^{134}$

Briefly, a relative risk is measured in prospective longitudinal cohort study. A group of disease free individuals are identified, the presence or absence of the relevant exposure (for example, smoking) is determined at baseline, and then the subjects are followed and the development of disease (for example, lung cancer) in exposed and non-exposed subjects is measured and compared. If, after correcting for any difference between the two groups in the length of follow up, the incidence rate of lung cancer is five times

Address for reprint requests: Dr Peter Sandercock, Department of Clinical Sciences, Western General Hospital, Crewe Road, Edinburgh EH4 2XU, UK.

Received 30 January 1989. Accepted 6 February 1989 greater in smokers than non-smokers, the relative risk is said to be five. ${ }^{134}$

However, cohort studies are expensive, take a long time to generate results and, though many individuals are recruited, a relatively small number will develop the disease under study. Case-control studies can be mounted much more quickly and cheaply; two groups of subjects are assembled-one known to have the disease (cases) and the other who are, as far as possible, known to be free of the disease but come from the same general population (controls). Both groups are examined for evidence of previous exposure. The methods used to identify, collect and examine cases and controls are much more important sources of error and bias than the statistical methods. ${ }^{134}$ Since the cases were assembled after they had developed signs of disease the incidence rate is unknown, so it is not possible to calculate relative risk. However, the ratio of the odds that a case had been exposed (a:c in the example in table 1) to the odds that a control had been exposed (b:d), algebraically the same as ad/bc, is a close approximation to the relative risk that would be obtained from a comparable cohort

Table 1 Case-control study: multiple sclerosis and sheep exposure

\begin{tabular}{|c|c|c|}
\hline & $\begin{array}{l}\text { Multiple Sclerosis } \\
\text { cases } \\
n=200\end{array}$ & $\begin{array}{l}\text { Healthy Controls } \\
n=200\end{array}$ \\
\hline $\begin{array}{l}\text { Shepherds } \\
\text { No sheep exposure }\end{array}$ & $\begin{array}{r}20^{a} \\
180^{c}\end{array}$ & $196^{d}$ \\
\hline
\end{tabular}


study, ${ }^{13}$ provided the incidence of disease is fairly low in exposed and non-exposed subjects (or, in randomised trials, only a small proportion of patients have outcome events). The calculation of confidence intervals for odds ratios is much simpler than for risk ratios.

The odds ratio is therefore-like the relative risk - a measure of association. In the example, the odds ratio was $5 \cdot 3$, which means that the odds of occupational exposure to sheep were 5.3 times higher in cases than controls. The odds ratio thus indicates the direction of the association; an odds ratio greater than one indicates a positive association between exposure and disease, odds ratio equal to one indicates no association, and the odds ratio less than one indicates a negative association. Furthermore, the odds ratio indicates the strength of the association; odds ratios close to one indicate only weak associations, whereas ratios over about three for positive associations, or near zero for negative associations indicate strong associations. Of course, the presence of a strong association is not proof of causation. Proof of causation generally depends on other factors such as consistency across several studies, specificity of the association, biological plausibility, and evidence of a dose-response relationship between exposure and the risk of developing the disease. ${ }^{134}$

It is a relatively simple procedure to calculate the approximate $95 \%$ confidence intervals for an odds ratio (Appendix). ${ }^{1-3}$ The confidence intervals immediately allow a test of statistical significance (at the $5 \%$ level for $95 \%$ confidence intervals); if the interval includes an odds ratio of one, the result is not significant. The interval also gives a feeling for the likely degree of sampling error; in the example given, although the confidence intervals do not include one and the difference between cases and controls is therefore significant, there is still a wide degree of uncertainty. The results would not only be compatible with only a very weak association (lower confidence limit odds ratio 1.8 ) but also a very strong one (upper confidence limit odds ratio $16 \cdot 2$ ).

The more traditional measure, the $\chi^{2}$ statistic, confirms a statistically significant result, but does not give any indication of the strength or direction of the

Table 2 Randomised controlled study: Cerebro protective agent in acute stroke

\begin{tabular}{lcc}
\hline & $\begin{array}{l}\text { Treated } \\
n=2000\end{array}$ & $\begin{array}{l}\text { Placebo } \\
n=2000\end{array}$ \\
\hline $\begin{array}{l}\text { Dead or disabled from stroke } \\
\text { Alive and not disabled }\end{array}$ & $\begin{array}{c}264(13 \cdot 2 \%) \\
1736\end{array}$ & $\begin{array}{c}400(20 \%) \\
1600\end{array}$ \\
\hline $\begin{array}{l}\text { Odds ratio }=0.61 . \\
\text { 95\% confidence interval } 0 \cdot 51-0.72 .\end{array}$ & $\begin{array}{l}\chi^{2}=32 \cdot 9 . \\
\text { Odds reduction }=39 \% .0 \cdot 0001 . \\
95 \% \text { confidence interval } 28 \% \text { to } 49 \% .\end{array}$ \\
\hline
\end{tabular}

association, nor of the likely sampling error. The confidence intervals in this example indicate that a larger sample would be required to determine the strength and directon of the association more precisely.

The advantage of the odds ratio over the $\chi^{2}$ becomes clearer if one considers a smaller study with, say, only 50 cases and 50 controls. If there were five shepherds amongst the cases and one shepherd amongst the controls, the odds ratio would be almost identical (5.4). The confidence intervals would be much wider $(0.6$ to 48.3$)$, indicating that there might be an association as strong as, and in the same direction as, the first example, but with a much larger sampling error because of a small sample size. The $\chi^{2}$ statistic $(1.60)$ is uninformative and merely shows no significant association.

The odds ratio and its $95 \%$ confidence interval are also useful in summarising the results of randomised controlled trials of treatments. In table 2, the results of a randomised controlled trial are given. In this case, the convention is to arrange the layout of the $2 \times 2$ table so that if active treatment is effective, and reduces the odds of the outcome event, in this case death or disability related to stroke, then the odds ratio (that is, the odds of being dead or disabled if allocated active treatment divided by the odds of being dead or disabled if allocated placebo) is less than one. An odds ratio of one would indicate no treatment effect: (11) $\times 100=0$. In the example, the odds ratio is 0.61 ; in other words treatment reduced the odds of death or disability by $(1-0.61) \times 100=39 \%$. The confidence interval in this example is narrow, so the most pessimistic estimate of treatment effect is that it only reduces the odds of death or disability by (1$0.72) \times 100=28 \%$ or alternatively, the reduction might be as much as $(1-0.51) \times 100=49 \%$. In either event the result is clearly statistically significant, since the confidence interval does not include one, but more important than mere statistical significance, the reduction is clinically significant too, even assuming the worst case of the upper confidence limit, of a $28 \%$ odds reduction.

The odds ratio also is useful in a statistical overview of several different randomised trials, because of its statistical robustness and simplicity of manipulation. ${ }^{5}$ The odds ratio and its $95 \%$ confidence interval are a summary of the results of a single trial and when displayed graphically alongside the other trials included in the overview, allow a rapid visual assessment of the results of each separate trial, any heterogeneity of treatment effect between trials and also the extent of random error. Thus, in an overview of trials of antiplatelet agents in patients with vascular disease, such a graphical display made it clear that many of the apparent differences between trials (gen- 
erally the smaller ones with wide confidence intervals) was more to do with the play of chance than real differences in treatment effect. ${ }^{5}$ This interpretation would not be possible in an analysis based on simple significance testing. ${ }^{5}$

The odds ratio may also be used in descriptive studies in which a group of cases has been assembled without any controls; the cases in the example, a study of patients with cerebral aneurysms (table 3a) have been divided into those with and without epilepsy. The odds ratio suggests post-operative epilepsy occurs $2 \cdot 7$ times more often amongst patients with middle cerebral artery (MCA) aneurysms but the width of the confidence intervals, coming close to one, emphasises the uncertainty about the strength of the association; it is, however, conventionally significant at the $5 \%$ level. If one of the $\mathbf{4 0}$ cases without post-operative epilepsy with a non-MCA aneurysm were to be excluded from the analysis (say because the angiogram had been reexamined and did not show an aneurysm) then the odds ratio and confidence intervals would change very little (table $3 \mathrm{~b}$ and figure), yet the $\chi^{2}$ statistic would no longer be significant. This illustrates the absurdity of choosing a single criterion of $p<0.05$ to differentiate "significant" from"non-significant". Is $p=0.0495$ so very different from $p=0.057$ ? Of course not, yet in many papers, the former would be reported as "significant" and the latter "non-significant"; by implication "clinically relevant" and "clinically irrelevant" respectively. Inspection of the confidence intervals reveals a clearer but statistically more uncertain picture, and graphical display reveals how similar the two results really are (fig).

Table 3 (a) Descriptive study: association between middle cerebral artery aneurysms and post-operative epilepsy in patients with subarachnoid haemorrhage

\begin{tabular}{lll}
\hline & $\begin{array}{l}\text { MCA } \\
\text { aneurysm } \\
n=50\end{array}$ & $\begin{array}{l}\text { Aneurysms } \\
\text { elsewhere } \\
n=50\end{array}$ \\
\hline $\begin{array}{l}\text { Epilepsy post-op } \\
\text { No epilepsy post-op }\end{array}$ & 20 & 10 \\
\hline Odds ratio = 2.7. & 30 & 40 \\
$95 \%$ confidence interval 1.1 to 6.5. & & $\begin{array}{l}\chi^{2}=3.86 . \\
2 \mathrm{p}=0.0495 .\end{array}$
\end{tabular}

(b) Reanalysis of data in (a) after one patient without epilepsy excluded from analysis

\begin{tabular}{lll}
\hline & $\begin{array}{l}\text { MCA } \\
\text { aneurysms }\end{array}$ & $\begin{array}{l}\text { Aneurysms } \\
\text { elsewhere }\end{array}$ \\
\hline $\begin{array}{l}\text { Epilepsy post-op } \\
\text { No epilepsy post-op }\end{array}$ & 20 & 10 \\
\hline $\begin{array}{l}\text { Odds ratio }=2 \cdot 6 . \\
95 \% \text { confidence interval 1.0 to 6.4. }\end{array}$ & 30 & 39 \\
\hline
\end{tabular}

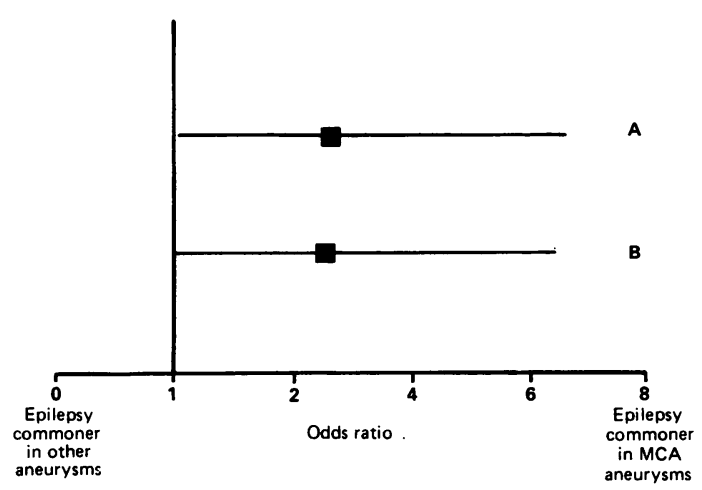

Fig The graph illustrates the data in tables $3 a$ and $3 b$. An odds ratio of one would suggest no association between aneurysm location and development of post-operative epilepsy. The square gives the point estimate of the odds ratio and the horizontal lines give the $95 \%$ confidence intervals. In this example, the odds ratio suggests that epilepsy occurs 2.6 times more often in patients with MCA aneurysms compared with other aneurysm sites. $A$ and $B$ correspond to tables $3 a$ and $3 b$. In $A$, the confidence interval does (not quite) include one, and the result is significant at the $5 \%$ level. In $B$, the confidence interval includes one and the result is nonsignificant.

One word of caution. In a graphical display, wide confidence intervals are more visually striking than narrow confidence intervals, yet greater emphasis should be placed on studies with narrow intervals because the result is more precise (that is, less sampling error). ${ }^{5}$ Peto has suggested that the point estimate of the odds ratio should be drawn as a black square whose size is proportional to the statistical power of the study; the bigger the power, the larger the square. ${ }^{5}$ This then visually offsets the effects of the associated short confidence interval in a powerful study.

In summary, the odds ratio has many properties which make it useful in expressing the results of a variety of different research designs employed in the neurosciences. The time for simple significance testing and tabulation of $\chi^{2}$ has long passed; estimation of the size and direction of effects, taking account of sampling error and the statistical power of the study are now necessary, and these requirements are fulfilled by the odds ratio and its $95 \%$ confidence interval.

\section{Appendix}

One fairly simple way to calculate a good approximation to the $95 \%$ confidence interval ${ }^{23}$ is:

First, calculate the standard error of the natural logarithm of the odds ratio $\left(\log _{e}\right.$ OR)

$$
S E\left(\log _{e} O R\right)=\sqrt{\frac{1}{a}+\frac{1}{b}+\frac{1}{c}+\frac{1}{d}}
$$




$$
=\sqrt{\frac{1}{20}+\frac{1}{4}+\frac{1}{180}+\frac{1}{196}}=0.56
$$

Next, calculate

$\mathrm{Y}=\log _{\mathrm{e}} \mathrm{OR}-\left(1.96 \times \mathrm{SE}\left(\log _{\mathrm{e}} \mathrm{OR}\right)\right)$

$Z=\log _{e} O R+\left(1.96 \times S E\left(\log _{e} O R\right)\right)$

$\left.Y=\log _{e} 5.4-1.96 \times 0.56\right)=0.60$

$Z=\log _{e} 5.4+(1.96 \times 0.56)=2.79$

The lower $95 \%$ confidence interval for the odds ratio is:

$\mathrm{e}^{\mathrm{y}}=\mathrm{e}^{0.60}=1.8$

And the upper $95 \%$ confidence interval is:

$\mathrm{e}^{\mathrm{z}}=\mathrm{e}^{2.79}=16 \cdot 2$

This approximation is less accurate in very small samples, especially if the odds ratio is close to unity, in which case, exact methods have to be used.

This formula is available as a programme listing, written in GW-BASIC, which runs on IBM-compatible computers or as a similar programme for the Texas
Instruments TI-66 programmable calculator; please send a self-addressed envelope to the author, if a copy is required.

\section{References}

1 Feinstein AR. Clinical Epidemiology. The Architecture of Clinical Research. Philadelphia: Saunders, 1985:420-34.

2 Morris J, Gardner M. Calculating confidence intervals for relative risks (odds ratios) and standardised ratios and rates. $\mathrm{Br} \mathrm{Med} \mathrm{J} \mathrm{1988;296:1313-36.}$

3 Breslow NE, Day NE. Statistical Methods in Cancer Research Vol I-The Analysis of case-control studies. IARC Publication no 32. Lyon: International Agency for Research on Cancer, 1980:42-81.

4 Sackett D, Haynes RB, Tugwell P. Clinical Epidemiology. A Science for Clinical Medicine. Toronto: Little Brown, 1985.

5 Antiplatelet trialists collaboration. Secondary prevention of vascular disease by prolonged antiplatelet treatment. Br Med J 1988;296:320-31. 